\title{
The source of polarized ions (SPI) and low energy polarimeter for the NUCLOTRON facility
}

\author{
V.V. Fimushkin ${ }^{1}$ \\ Joint Institute for Nuclear Research \\ Joliot-Curie 6, 141980 Dubna, Moscow region, Russia \\ E-mail: fimushkin@jinr.ru
}

R.A. Kuzyakin, M.V. Kulikov, L.V. Kutuzova, Yu.V. Prokofichev, A.M. Shumkov

Joint Institute for Nuclear Research

Joliot-Curie 6, 141980 Dubna, Moscow region, Russia

\section{A.S. Belov, A.V. Turbabin, and V.N. Zubets}

Institute for Nuclear Research of RAS

Prospect 60letiya Oktyabrya, 7A, 117312, Moscow, Russia

\begin{abstract}
The paper describes the JINR polarized ion source operating by means of the atomic beam method. The latest results of the SPI testing are presented. Polarized and unpolarized deuteron beams as well as the polarized proton beam were produced to accelerate in the NUCLOTRON. The conceptual design of a new universal low energy polarimeter at NUCLOTRON is also presented. It is proposed to install a new polarimeter behind the linear accelerator. The ${ }^{3} \mathrm{He}$ target of this setup allows one to carry out measuring both the vector polarization of protons and vector and tensor polarization of deuterons. It is assumed that the new design of the polarimeter will make it possible to measure vector and tensor polarization of deuterons at the same time. Fabrication of the basic parts of the modern polarimeter is in progress now.
\end{abstract}

23rd International Spin Physics Symposium - SPIN2018 -

10-14 September, 2018

Ferrara, Italy

\section{${ }^{1}$ Speaker}




\section{Introduction}

The high intensity pulsed source of polarized ions (SPI) has been developed at JINR in collaboration with INR RAS for injection of polarized deuterons and protons into the NUCLOTRON and future collider of heavy and light ions NICA [1]. The SPI is an atomic beam-type polarized ion source with a charge-exchange plasma ionizer and a storage cell in the ionization region [2-9].

\section{Source of polarized ions}

\subsection{General description of the SPI}

A schematic diagram and a general view of the SPI are shown in Figures 1 and 2. The source consists of an atomic beam apparatus, a plasma charge-exchange ionizer and a system of transporting the polarized ion beam and turn the spin to the vertical direction.

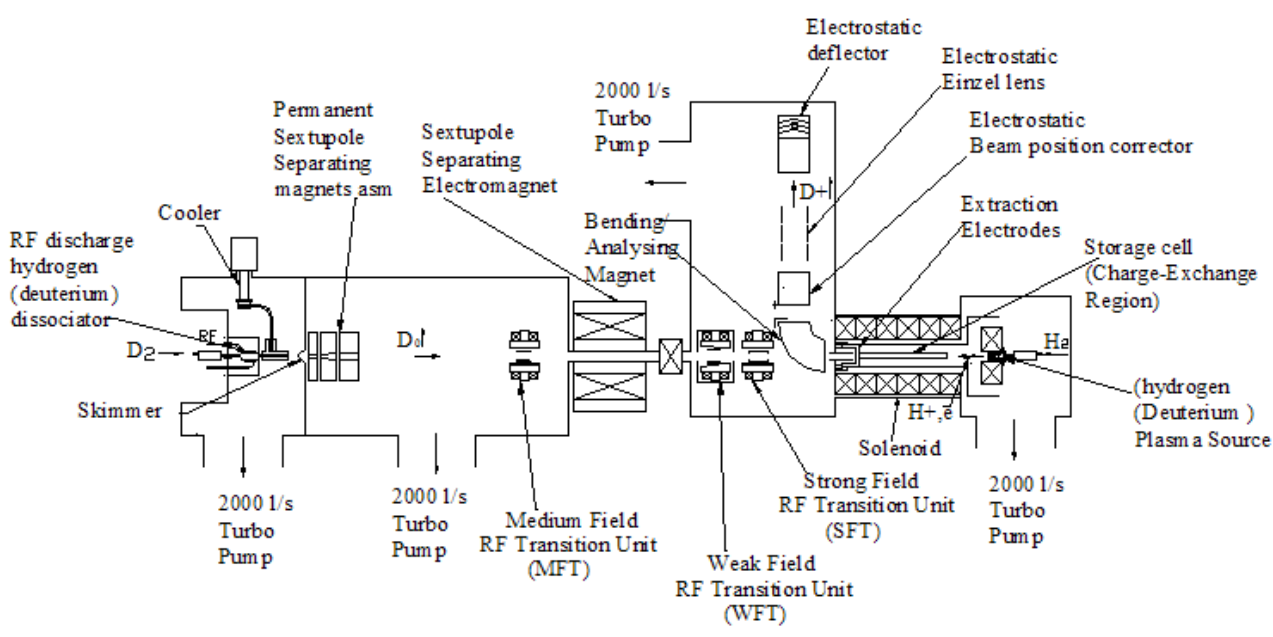

Figure 1. Schematic diagram of the SPI.

The test bench installation setup is shown in Figure 2.

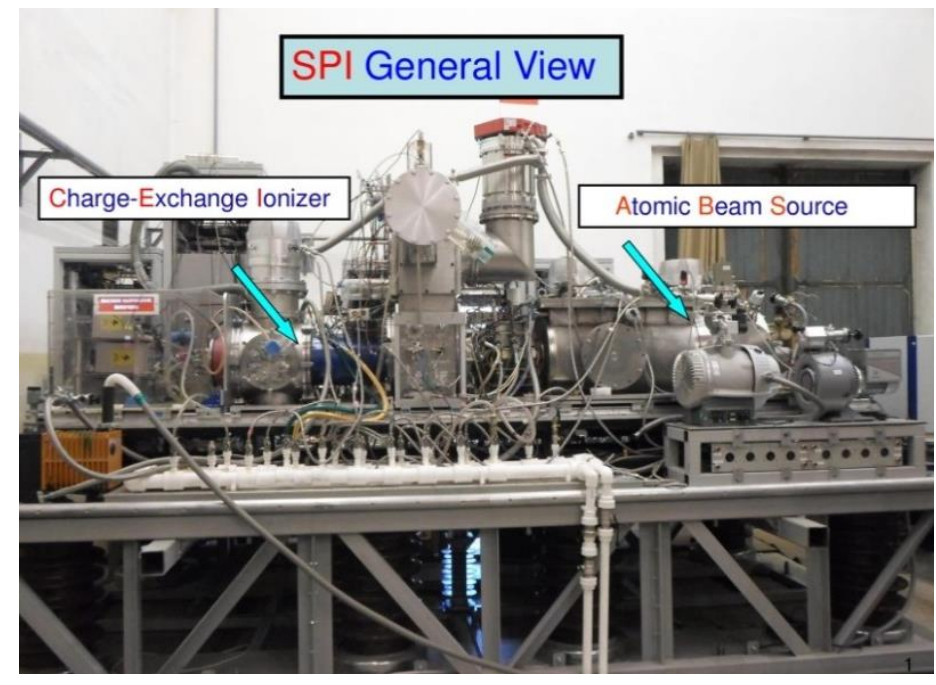

Figure 2. SPI general view. 
When carrying out the runs with the polarized beams, the SPI is located on the high voltage platform and joint with the preaccelerator according to the scheme shown in Figures 3 and 4. The data obtained from the SPI remote control and management systems are readout from the high voltage platform of the Lu-20 linac by means of the fibre-glass system to the linac control room.

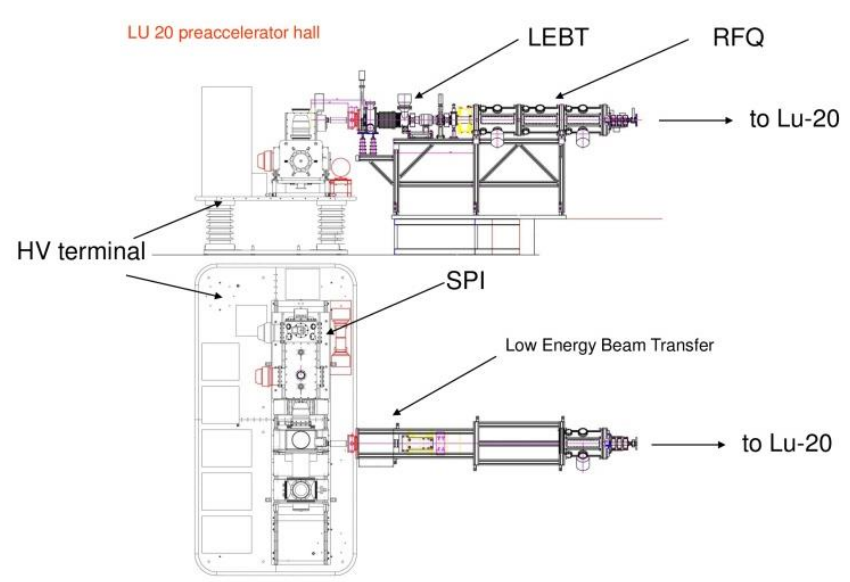

Figure 3. SPI, LEBT, RFQ layout at linac Lu-20.

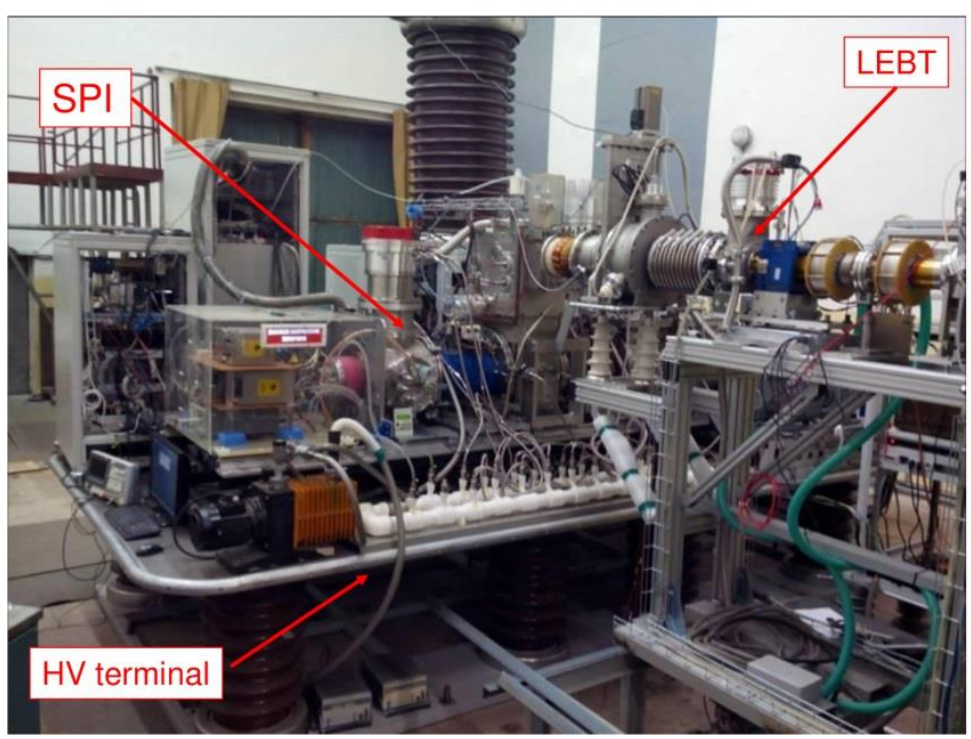

Figure 4. SPI, LEBT general view at Lu-20 preaccelerator hall (operational assembly).

\subsection{Tests of the SPI}

After successful runs with the polarized beams of deuterons and protons at the NUCLOTRON in 2016-2017 [10-12], the work to achieve the SPI design parameters is continued. The goal of the project is to obtain polarized beams of deuterons and protons at the output of the source up to $10 \mathrm{~mA}$.

At present we have managed to increase the current of the deuteron polarized beam of the SPI facility up to $6 \mathrm{~mA}$, Figure 5. 


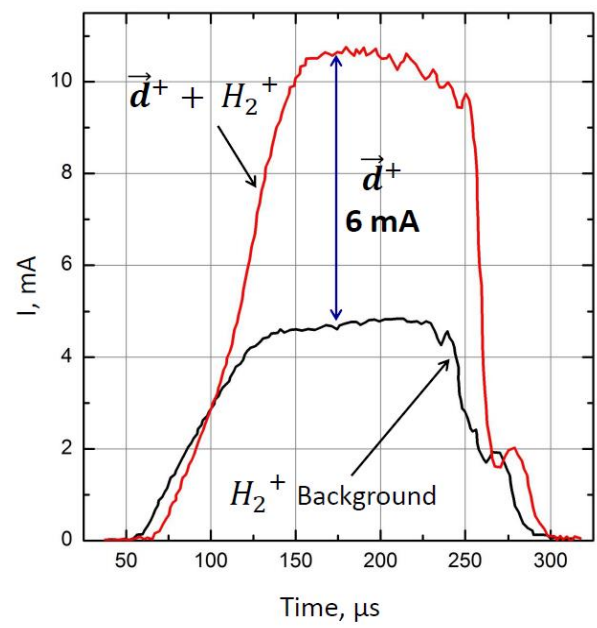

Figure 5. Polarized deuteron current downstream the analyzing magnet.

The result has been obtained due to optimization of the configuration of the solenoid magnetic field of the ionizer, improvement of the storage cell construction, updating of the ion beam extraction system of the charge-exchange ionizer and increase of the atom beam intensity with the additional sextupole magnet.

\subsection{Nearest plans}

Further reducing of the $\mathrm{H}_{2}{ }^{+}$background assumes testing of new versions of the storage cell. The optimal structure of the ion-optical transporting system of the strong-current polarized beam inside the SPI is developed. We are about to complete the work of upgrading the Weak Field RF Transition Unit for the following increase of the polarization degree of the polarized beams of deuterons and protons. In 2019 it is planned to finish the development of the new low energy polarimeter.

\section{Low energy polarimeter}

Low energy polarimeter (LEP) is the first of the three polarimeters of polarized beams at the NUCLOTRON accelerator complex. This polarimeter is placed behind the linear accelerator Lu-20. Its operating energy is $5 \mathrm{MeV}$ per nucleon. The main advantage of this polarimeter is that the setup may be used not only for polarization measuring but also for tuning SPI RF transition units (WFT, MFT, SFT). So, it is an important task to have an up-to-date working low energy polarimeter.

In Figure 6 the conceptual design of a new universal low energy polarimeter is presented. The ${ }^{3} \mathrm{He}$ target of this setup allows one to measure both the vector polarization of protons (with ${ }^{3} \mathrm{He}(\mathrm{p}, \mathrm{p})^{3} \mathrm{He}$ elastic scattering reaction) and the vector and tensor polarization of deuterons (with ${ }^{3} \mathrm{He}(\mathrm{d}, \mathrm{d}){ }^{3} \mathrm{He}$ elastic scattering reaction and ${ }^{3} \mathrm{He}(\mathrm{d}, \mathrm{p}){ }^{4} \mathrm{He}$ nuclear reaction). The main features of the new LEP are:

- Up-to-date fast detectors will be used. It is assumed to measure the polarization of each bunch.

- The data flow will be protected from electromagnetic noises.

- It is assumed that the design of the polarimeter will make it possible to measure vector and tensor polarization of deuterons with one setup at the same time (It is necessary 
because SPI can produce beams polarized in different tensor and vector modes at the same time).

- The dipole magnet will be used to remove $\mathrm{H}_{2}{ }^{+}$component in deuteron beam. After accelerating in the linear accelerator Lu-20 and passing through a stripping target the unpolarized protons are formed due to stripping of the $\mathrm{H}_{2}{ }^{+}$ions. These protons will be removed by a dipole magnet.

- The set-up design will allow us to control the polarization at inter cycle switch-on.

The polarized deuteron or proton beam coming from the linear accelerator Lu-20 goes through the vacuum gate to the dipole magnet. Then going through a movable Faraday cup it comes to the detector vacuum chamber.

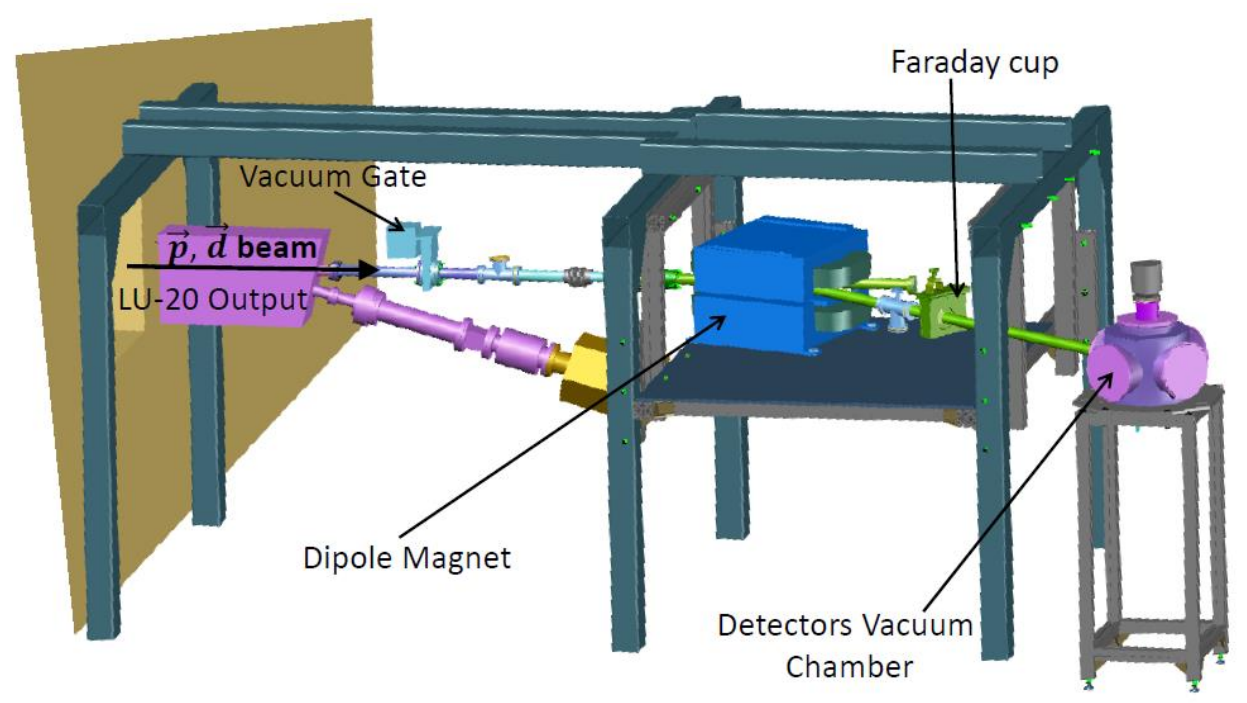

Figure 6. General view of the new universal low energy polarimeter behind the linear accelerator Lu-20.

In Figure 7 the detector assembly concept for the LEP is shown. The detectors will be placed in the vacuum chamber. It is assumed to use silicon detectors with $20 * 20 \mathrm{~mm}^{2}$ size and $300 \mu \mathrm{m}$ thickness. The detectors at a distant plate would be used for the deuteron beam and detectors on a substrate for proton beam polarization measuring. This detector scheme allows one to measure not only the flat but spatial beam polarization. All the assembly is placed on one flange that is rather handy.

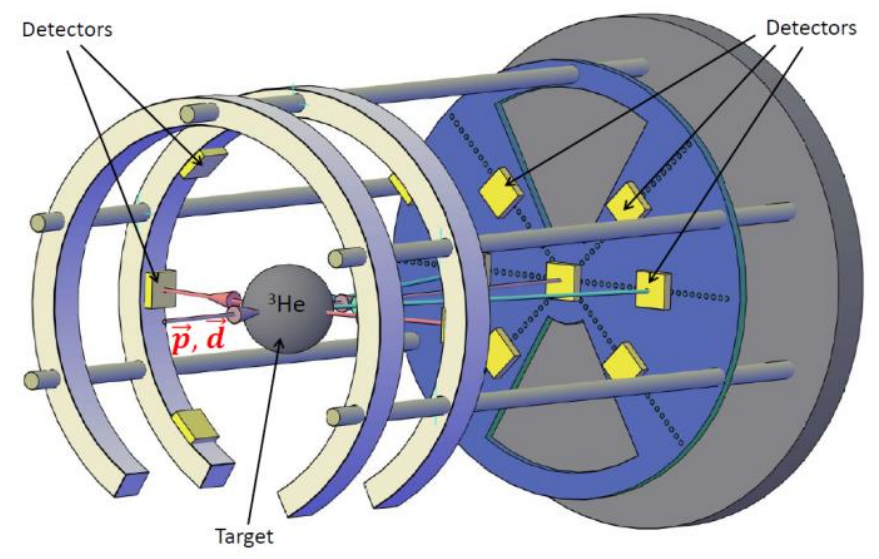

Figure 7. Detectors assembly for the new universal low energy polarimeter. 
Fabrication of the basic parts of the modern polarimeter is in progress now. It is supposed to test the setup in 2019 .

\section{Conclusions}

The SPI development is coming to its completing stage. The measurements of the polarization degree of the polarized beams of deuterons and protons of low energy will promote efficient carrying out polarization studies at the NUCLOTRON.

\section{References}

[1] G.V. Trubnikov, N.N. Agapov, O.I. Brovko, A.V. Butenko, E.D. Donets, A.V. Eliseev, V.V. Fimushkin et al., Proc. of the $4^{\text {th }}$ Int. Particle Accelerator Conf. IPAC2013, TUPFI009 1343 (2013).

[2] A.S. Belov, S.K. Esin, S.A. Kubalov, V.E. Kuzik, A.A. Stepanov, and V.P. Yakushev, Nucl. Instrum. and Meth. In Phys. Res. A255 (1987) 442.

[3] A.S. Belov, S.K. Esin, L.P. Netchaeva, V.S. Klenov, A.V. Turbabin, and G.A. Vasil'ev, Proc. Int. Workshop on Polarized Gas Targets and Polarized Beams, AIP Conf. Proc. 421 (1998) 362.

[4] A.S. Belov, S.K. Esin, L.P. Netchaeva, A.V. Turbabin, and G.A. Vasil'ev, Proc. Int. Symp. on High Energy Spin Physics, (1999) 22.

[5] V.P. Derenchuk and A.S. Belov, Proc. 2001 Particle Accelerator Conf., WPAH008 2093 (2001).

[6] V.V. Fimushkin, A.D. Kovalenko, L.V. Kutuzova, Yu.V. Prokofichev, A.S. Belov, A.V. Turbabin, and V.N. Zubets, Proc. 20th Int. Symp. on High Energy Spin Physics, PEPAN 45 (2014) 297.

[7] V.V. Fimushkin, A.D. Kovalenko, L.V. Kutuzova, Yu.V. Prokofichev, A.S. Belov, A.V. Turbabin, and V.N. Zubets, XVI Workshop on High Energy Spin Physics, IOP Conf. Ser. 678 (2016) 012058.

[8] A.S. Belov, D.E. Donets, V.V. Fimushkin, A.D. Kovalenko, L.V. Kutuzova, Yu.V. Prokofichev, V.B. Shutov, A.V. Turbabin, V.N. Zubets, XVII Workshop on High Energy Spin Physics, IOP Conf. Ser. 938 (2017) 012017.

[9] V.V. Fimushkin, A.D. Kovalenko, R.A. Kuzyakin, M.V. Kulikov, L.V. Kutuzova, Yu.A. Plis, Yu.V. Prokofichev, V.B. Shutov, A.S. Belov, A.V. Turbabin, and V.N. Zubets, PoS 324 (2018) 019.

[10] Y. Skhomenko, V.P. Ladygin et al., XVII Workshop on High Energy Spin Physics, IOP Conf. Ser. 938 (2017) 012022.

[11] V.P. Ladygin, Yu.V. Gurchin et al., XVII Workshop on High Energy Spin Physics, IOP Conf. Ser. 938 (2017) 012008.

[12] Yu.N. Filatov, A.V. Butenko, A.D. Kovalenko, V.A. Mikhaylov, A.M. Kondratenko, M.A. Kondratenko, XVII Workshop on High Energy Spin Physics, IOP Conf. Ser. 938 (2017) 012018 . 